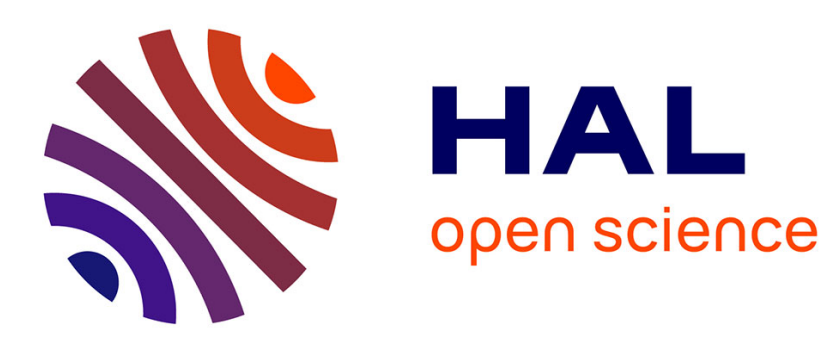

\title{
Reversible magnetic clamp of a microfluidic interface for the seric detection of food allergies on allergen microarrays
}

Julie Foncy, Erwan Crestel, Jean-Philippe Borges, Aurore Estève, Jean Christophe Cau, Christophe Vieu, Laurent Malaquin, Emmanuelle Trévisiol

\section{To cite this version:}

Julie Foncy, Erwan Crestel, Jean-Philippe Borges, Aurore Estève, Jean Christophe Cau, et al.. Reversible magnetic clamp of a microfluidic interface for the seric detection of food allergies on allergen microarrays. Microelectronic Engineering, 2016, 158, pp.16 - 21. 10.1016/j.mee.2016.03.005 . hal01484322

\author{
HAL Id: hal-01484322 \\ https://hal.laas.fr/hal-01484322
}

Submitted on 7 Mar 2017

HAL is a multi-disciplinary open access archive for the deposit and dissemination of scientific research documents, whether they are published or not. The documents may come from teaching and research institutions in France or abroad, or from public or private research centers.
L'archive ouverte pluridisciplinaire HAL, est destinée au dépôt et à la diffusion de documents scientifiques de niveau recherche, publiés ou non, émanant des établissements d'enseignement et de recherche français ou étrangers, des laboratoires publics ou privés. 


\title{
Reversible magnetic clamp of a microfluidic interface for the seric detection of food allergies on allergen microarrays
}

\author{
Julie Foncy ${ }^{\mathrm{a}}{ }^{\ddagger}$, Erwan Crestel $^{\mathrm{b}}{ }^{\ddagger}$, Jean-Philippe Borges ${ }^{\mathrm{a}}$, Aurore Estève ${ }^{\mathrm{a}}$, Jean Christophe Cau \\ b, Christophe Vieu ${ }^{\mathrm{a}, \mathrm{c}}$, Laurent Malaquin ${ }^{\mathrm{a}}$, Emmanuelle Trévisiol ${ }^{\text {a,d,e,f }}$ \\ ${ }^{a}$ CNRS, LAAS, 7 Avenue du Colonel Roche, F-31400 Toulouse, France \\ ${ }^{\mathrm{b}}$ Innopsys, Parc d'Activités Activestre, 31390 Carbonne, France \\ ${ }^{\mathrm{c}}$ Univ de Toulouse, INSA, LAAS, F-31400 Toulouse, France \\ ${ }^{\mathrm{d}}$ Université de Toulouse; INSA, UPS, INP; LISBP, 135 Avenue de Rangueil, F-31077 Toulouse, France \\ e INRA, UMR792 Ingénierie des Systèmes Biologiques et des Procédés, F-31400 Toulouse, France \\ ${ }^{\mathrm{f}}$ CNRS, UMR 5504, F-31400 Toulouse, France
}

Corresponding author: e-mail: julie.foncy@laas.fr

‡: These authors contributed equally to the work

Keywords: microfluidics, reversible magnetic clamp, multiplexed immunoassay, allergen microarray

\begin{abstract}
To provide a robust platform for fluid handling, most microfluidic devices usually involve irreversible bonding methods to achieve a leak free interface between the microchannels and the holding substrate. Such an approach induces a major drawback when biological interactions are performed on a microarray format as it is difficult to recover the biochip for further fluorescence scanner analysis. This work describes an automated microfluidic platform using a reversible magnetic clamp for multiplexed immunodiagnostis. The microfluidic device is composed of a magnetic PDMS layer (containing iron powder) coated by PDMS, which is reversibly clamped to an epoxysilane glass slide containing an array of various antigens. The microfluidic device was validated for in vitro diagnosis of food allergies on an allergen microarray after serum interaction. The statistical analysis of spot intensities
\end{abstract}


(Signal to noise ratios) on the microarray displayed excellent reproducibility. In addition to the reduction of volumes provided by miniaturization, this approach is versatile, easy-toproduce and provide an effective platform for multiplexed immunodiagnosis based on conventional fluorescent detection schemes. 


\section{Introduction}

Immunoassays are routine tests mainly devoted to in vitro diagnosis and biological or pharmaceutical R\&D. They exploit the specificity of antigen-antibody recognition for the detection of biological analytes of interest in patient samples [1]. The best-known assay is the enzyme-linked immunosorbent assay (ELISA) in which the detection of an antigen-antibody complex is performed on a microtiter plate [2]. Most of the analytical methods currently used for clinical diagnosis involve manual processing that, in the most detrimental cases, might be the sources of contamination, non-reproducible errors that lead to false positive or negative results. In this context, microfluidic systems have demonstrated many advantages to improve the reproducibility of bioanalytical protocols while providing additional advantages regarding miniaturization, integration and automation [3]. Bringing together microfluidics and immunoassays lead to promising tools for automatic, sensitive and selective measurements of biological components.

Ensuring the compatibility of microfluidic devices with well-established biological methods and standard substrates is essential in the development of novel analytical platforms. This is particularly the case of microarray technologies that cover a large range of applications in biomolecular analysis. Indeed, in order to provide a robust platform for fluid handling, most microfluidic devices usually involve irreversible bonding methods to achieve a leak-free interface between the microchannels and the holding substrate. Such approach give rise to a major drawback when biological interactions are performed on a microarray format as it is difficult to recover the biochip for further fluorescence scanner analysis. Several methods to reversibly seal microfluidic device were described in the literature, including sealing by selfadhesion properties of the material, vacuum seal by aspiration or sealing by magnetism. Each process has advantages and drawbacks regarding implementation, cost and target applications. The sealing by self-adhesion is described to be weak. Hence, surface treatment need to be achieved to enhance the contact strength between the microfluidic device and the substrate [4]. On another side, the reversible clamping directed by vacuum requires complex setups and the addition of a microchannel network for the sealing by aspiration of the substrate and the microfluidic device [5]. Finally, the sealing by magnetism is described to be easy to implement, biocompatible and inexpensive [6-8].

Here, we describe the design, fabrication and validation of a generic microfluidic interface allowing multiplexed detection of food allergies on a biochip format using a reversible magnetic clamp. The microfluidic device is composed of a magnetic 
polydimethylsiloxane (PDMS) layer (containing iron powder), which is reversibly sealed to an epoxysilane glass slide containing an array of food allergens. The channel design integrates two independent inlets to separate the required buffers (saturation and washing buffers, labelled secondary antibody) from sample (serum, blood) and avoid potential contaminations during the sequential injections. Despite the integration of magnetic particles within the microfluidic chip, the fabrication process was optimized to permit optical observations during the analytical workflow. The geometry and dimensions of the channels were optimised through hydrodynamic simulations performed with COMSOL software to provide a uniform spreading of the reactant and analytes on the capture area. The functionality of the device was validated for in vitro diagnosis of food allergies on a microarray of spotted allergens after interaction with the serum of a well characterized patient.

\section{Material and methods}

\section{Microfluidic device design and fabrication}

The microfluidic design (Fig. 1a) consists of a y-shape structure comprising two independent inlets (one for injecting buffers and secondary labelled antibody solutions and the other for serum injection) connected to a larger incubation chamber (15 mm long x $1.5 \mathrm{~mm}$ wide x 0.2 $\mathrm{mm}$ high) where the capture spots are located. The geometry and dimensions of the three channels between the inlets and outlet were optimized through hydrodynamic simulations using COMSOL Multiphysics software. The differences in the channel dimensions were designed to reduce risks of contaminations by diffusion between the serum and the other liquids needed for the sequential biological protocol of interaction. Reduction of the dead volumes, and calculation of the hydrodynamic resistance of the devices were performed in order to ensure that required pressures and flow rates be compatible with the external liquid handling systems (see section below). The width in the main incubation chamber was also set to provide a uniform spreading of the reactants according to the spot distribution in the microarray.

The aluminium master mold (aluminium 2017A) required for the microfluidic device manufacturing was micro-machined using a 500- $\mu \mathrm{m}$-diameter mechanical tool. The microfluidic device is composed of one layer of magnetic PDMS covered by a layer of native PDMS (Fig. 1b). It was obtained in two steps. PDMS prepolymer and curing agent (Sylgard 184, Dow Corning) were mixed manually in a 10:1 ratio and iron powder (Sigma Aldrich, hydrogen reduced, $50 \mu \mathrm{m}$ diameter, 50/50, w/w) was further added to the PDMS prepolymer 
mixture. The magnetic PDMS prepolymer solution was degassed, carefully poured onto the master and cured for 1 hour at $65^{\circ} \mathrm{C}$ leading to a layer of $200 \mu \mathrm{m}$ height of magnetic PDMS. A doctor blade technique was used to remove excess of magnetic PDMS and provide uniform layer, with no material left on top of the protruding structures of the master mold. Then a second layer of PDMS, without iron powder, was casted on the top of the magnetic layer (12 h, $65^{\circ} \mathrm{C}$ ) (Fig. 1b). Finally, the PDMS microfluidic device was removed from the mold and inlets and outlet were punched.

\section{Allergen array manufacturing and serum}

Total food extracts (egg white and yolk, crab sticks, cashew nut, wheat, salmon, bass, cod, tuna, shrimp, cow milk, goat milk, peanut, mustard, buckwheat, sesame, soya, hazelnut, walnut) used in this study and sera of rabbits immunized against peanut were obtained from Pharma-DEV lab (AOMC team, Toulouse). The food extracts were dissolved at a concentration of $0.5 \mathrm{mg} / \mathrm{mL}$ in phosphate buffer ( $\left.25 \mathrm{mM} \mathrm{NaH}_{2} \mathrm{PO}_{4}, \mathrm{pH} 7.4\right)$ and were spotted in triplicate on epoxysilane slide (Nexterion ${ }^{\circledR}$ Slide E, Schott Nexterion, Jena, Germany) using a conventional microarrayer (Q-Array Mini, Genetix) equipped with pins (SMP3, ArrayIt) delivering around $2 \mathrm{~nL}$ of food extract solutions per spot with a medium size of 150 $\mu \mathrm{M}$ at $300 \mu \mathrm{M}$ pitch. The quality of the spotting was optimal at $45 \%$ relative humidity and constant temperature of $20^{\circ} \mathrm{C}$. After deposition food allergen microarrays were dried overnight under ambient conditions.

Human serum were obtained from Rangueil Hospital (Toulouse). Their specific IgE level were determined in serum by in vitro measurement of allergen-specific $\operatorname{IgE}$ by the ImmunoCAP ${ }^{\circledR}$ assay [9] (Thermo Scientific). For the patient tested in this study, the values determined by ImmunoCAP ${ }^{\circledR}$ specific IgE were cow and goat milk, $100 \mathrm{kUA} / \mathrm{L}$, egg yolk $13,8 \mathrm{kUA} / \mathrm{L}$ and peanut $4 \mathrm{kUA} / \mathrm{L}$.

\section{Microfluidic driven interactions on allergen array}

The magnetic PDMS microfluidic interface was manually aligned and brought into contact with the allergen array. Reversible clamp of the microfluidic device with the biochip was obtained after positioning magnets (4x8 magnets, NeodymeFerbore) beneath the microarray glass side (Fig.2). The microfluidic interface was then connected to a pressure-based flow rate controller (MFCS-EZ, 0-350 mBar, Fluigent) using polytetrafluoroethylene (PTFE) tubes (inner diameter: $1 \mathrm{~mm}, 0.56 \mathrm{~mm}$ and $0.3 \mathrm{~mm}$ ) for the injection of all reagents and a flowmeter (0-50 $\mu \mathrm{L} / \mathrm{min}$ range) was placed at the oulet to retro-control the flow rate (Fig. 3). 
Electromagnet switch valves (Fluigent) were inserted in between the reactant reservoirs and the corresponding device inlet. There were activated in between each reactant injection to isolate the unused reservoirs from the injection inlet. All injections were performed at a constant flow rate of $35 \mu \mathrm{L} / \mathrm{min}$. The procedure is described as follows. First, the buffer (phosphate saline buffer, PBS 1x, $10 \mathrm{mM}$ phosphate, $\mathrm{NaCl} 150 \mathrm{mM}$, tween $200.5 \% \mathrm{v} / \mathrm{v}$, PBST buffer) was injected and followed by passivation solution (Bovine serum albumin (BSA) or human serum albumin (HSA), $1 \%(\mathrm{v} / \mathrm{v})$ in PBST buffer, $50 \mu \mathrm{L})$. After incubation (5 min. at room temperature (RT)) the channel was rinsed with PBST and filled with $70 \mu \mathrm{L}$ of rabbit or human serum. The flow was stopped for $30 \mathrm{~min}$ to allow for the serum incubation (RT). Washing was performed with PBST buffer, and secondary labeled antibody $(80 \mu \mathrm{L}, 0.5$ $\mu \mathrm{g} / \mathrm{mL}$, anti-rabbit IgG-DyLight 649 or anti-human IgE labeled with allophycocyanin (APC) was injected, left to incubate during 15 minutes without flow and the microfluidic channel was then rinsed $(2 \times 1 \mathrm{~min})$ by injection of PBST. The microfluidic interface was finally emptied by injection of air and dried with a nitrogen flow. The magnets were removed allowing the release of the allergen microarray, which was scanned at a wavelength of $635 \mathrm{~nm}$ using InnoScan 1100 (Innopsys, Carbonne, France). Images were analyzed using Mapix software and the fluorescence intensities of each spot were calculated as the median of fluorescence signal.

\section{Results and discussion}

Manual processing of microscope glass slide microarrays is most common, sample and washing buffer being injected by an operator leading to irreproducible fluctuations, which could affect the quality and consistency of the final data. To increase reproducibility and develop a reliable molecular diagnostics assay, automation of these manual steps needs to be implemented. The aim of this work was to design and validate a microfluidic interface capable of managing all the analytical steps needed in any protein microarray interaction protocol.

\subsection{Fluid handling in the microfluidic interface}

First experiments to validate the effectiveness of the microfluidic platform to achieve complex analytical workflow including several injection and incubation steps, were performed by injecting water and dyes with varying pressures and monitoring the resulting 
flow rate in the device. We first investigated the maximum injection pressure that the magnetic clamping strategy may tolerate during the analysis. No leakage was observed below 150 mbar for both the reactants and serum injection channels. Of course, this value is very dependant on the channel geometry and it should not be taken as reference at it is directly related to the width and proximity of the channels in the devices. The use of a thicker magnetic PDMS layer is a simple and straightforward way of reaching higher-pressure values if necessary. It is also worth noticing that we did not observe channel collapse that might arise from the magnetic clamp force.

In the frame of our application this maximum pressure value corresponds to a flow rate close to $70 \mu \mathrm{L} / \mathrm{min}$ in the main capture chamber when considering the reactants inlet. This is well above the targeted values for this application (around $25 \mu \mathrm{L} / \mathrm{min}$ ). Investigation of the volumes required to perform an efficient filling of the main reservoir was also investigated. Taking into account the whole microfluidic device, we found that a volume of $18 \mu \mathrm{L}$ was sufficient to fill the incubation chamber and perform the interaction. These results were in good agreement with simulation (results not shown).

From the technological point of view, this approach is very appealing. Indeed, whereas conventional processes usually involve oxygen plasma treatment to provide a leakage free bonding in PDMS devices, this technology provide a robust and flexible strategy to integrate microfluidic on substrates, with almost no limitation in the size and material to be considered. It is of particular importance when considering bio-fonctionalized surfaces such as those used in this study where the use of any surface treatment or material deposition would be detrimental regarding the biological interaction. Another advantage provided by this technology is the possibility to perform optical imaging in the microfluidic device. Indeed, the doctor blade technology, permit to keep the device transparent above the microfluidic channels. This feature is advantageous for the alignment step and for the monitoring of fluid handling, but it also provides the possibility to perform analysis through transmission microscopy.

\subsection{Validation of microfluidic interactions on glass slides}

Before testing the capability of the microfluidic interface to detect specific IgE from human serum, we first validated all the parameters using rabbit serum. Peanut extract was spotted on epoxysilane glass slide ( 3 columns, 22 rows) and incubated, from 5 to 60 minutes, with serum containing IgG from rabbit immunized against peanut. The interactions were revealed after 
incubation with a secondary fluorescently labeled anti-rabbit IgG. Fig. 4b shows the image obtained after a complete protocol of interaction. All the spots were uniformly detected (red circles) whatever their localization in the channel and the background was very low, validating the passivation, interaction and washing processes. The optimal interaction time was found to be around 30 minutes for the serum and 15 minutes for the secondary labeled antibody. These parameters were directly used as a reference to perform interactions with human serum.

\subsection{Food allergies detection on allergen array using the magnetic microfluidic interface}

Food allergen extracts were deposited in triplicate (3 columns, 19 rows) and serum samples from allergic patients were screened for allergen-specific IgE determination. PBS buffer was also spotted and used as negative control. In case of positive matching between the antibodies from the patient's serum and the immobilized proteins on the array surface, a fluorescent signal was detected after injection of an anti-IgE secondary antibody, labeled with allophycocyanin (APC), allowing for the detection of allergen-specific IgE. All the buffers, serum and secondary antibody solutions were handled using the microfluidic interface reversibly clamped on the microarray surface by magnetic force without noticing any leakage in the system. Fig. 5a shows the image obtained after incubation of serum from a patient allergic to cow and goat milk as well as egg yolk. As it can be seen, negative control and nonallergenic proteins did not display any significant detectable fluorescent signal whereas fluorescent spots were clearly detectable in the expected area (Fig. 5a, cow milk, goat milk and egg yolk). After analysis of the microarray and processing using Mapix software, the signal levels from each allergen was retrieved and the signal-to-noise ratio (SNR) value was calculated (Fig. 5b). For microarrays, the SNR could help to distinguish whether the signal is significant or whether it is too weak to be considered for quantification. We set a SNR threshold value greater than 3 to be significant, leading to results in accordance with ImmnuoCAP ${ }^{\circledR}$ specific-IgE test.

\section{Conclusion}

We developed an original concept for the magnetic clamping of microfluidic devices. This method provides a simple and versatile way of integrating microfluidics with most substrates used in bioanalytical methods. The use of magnetic force is advantageous as it preserves the 
device functionality and does not require any specific surface treatments. We could demonstrate that the injection maximum pressure in the microfluidic chip is around 150 mbar, leading to flow rates close to $70 \mathrm{uL} / \mathrm{min}$ which is sufficient for most biological applications. The device was validated for an in vitro immunodiagnosis assay to detect food allergies in the serum of a human patient with a microarray of allergen spots. In addition to the reduction of volumes provided by miniaturization, this approach is versatile, easy-to-produce and provides an effective and automated platform for multiplexed immunodiagnosis that could be easily extended to DNA hybridization assays on solid supports. These promising results will be further consolidate on large cohort of patients. Beyond this application we believe that the magnetic clamp concept can be easily adapted to different substrate formats and favour the spreading of microfluidic technologies in analysis and diagnostic applications.

\section{Acknowledgments}

This work was supported by the National Research Agency (Agence Nationale pour la Recherche, "LABCOM" program (ANR-13-LAB2-0009-01). We thank Lidwine Trouilh from the GeT-Biochip Platform Toulouse Genopole for allergen spotting, Annick Barre and Pierre Rougé from Pharma-Dev Lab, AOMC team, for providing total food allergen extracts and Pol-André Apoil from Rangueil Hospital for supplying human serum. 
[1] M. Hartmann, J. Roeraade, D. Stoll, M.F. Templin, T.O. Joos, Protein microarrays for diagnostic assays, Anal Bioanal Chem, 393 (2009) 1407-1416.

[2] R.M. Lequin, Enzyme immunoassay (EIA)/enzyme-linked immunosorbent assay (ELISA), Clin Chem, 51 (2005) 2415-2418.

[3] C. Rivet, H. Lee, A. Hirsch, S. Hamilton, H. Lu, Microfluidics for medical diagnostics and biosensors, Chemical Engineering Science, 66 (2011) 1490-1507.

[4] J.C. McDonald, G.M. Whitesides, Poly(dimethylsiloxane) as a material for fabricating microfluidic devices, Acc Chem Res, 35 (2002) 491-499.

[5] M. Le Berre, C. Crozatier, G.V. Casquillas, Y. Chen, Reversible assembling of micorfluidic devices by aspiration, Microelectronic Engineering, 83 (2006) 1284-1287.

[6] E. Tkachenko, E. Gutierrez, M.H. Ginsberg, A. Groisman, An easy to assemble microfluidic perfusion device with a magnetic clamp, Lab Chip, 9 (2009) 1085-1095.

[7] R. M., F. Piraino, N. Sadr, M. Laganà, A. Redaelli, M. Moretti, Reliable magnetic reversible assembly of complex microfluidic devices: fabrication, characterization, and biological validation, Microfluidics and Nanofluidics, 10 (2010) 1097-1107.

[8] M. Rafat, D.R. Raad, A.C. Rowat, D.T. Auguste, Fabrication of reversibly adhesive fluidic devices using magnetism, Lab Chip, 9 (2009) 3016-3019.

[9] S.G. Johansson, ImmunoCAP Specific IgE test: an objective tool for research and routine allergy diagnosis, Expert Rev Mol Diagn, 4 (2004) 273-279.

Figures:

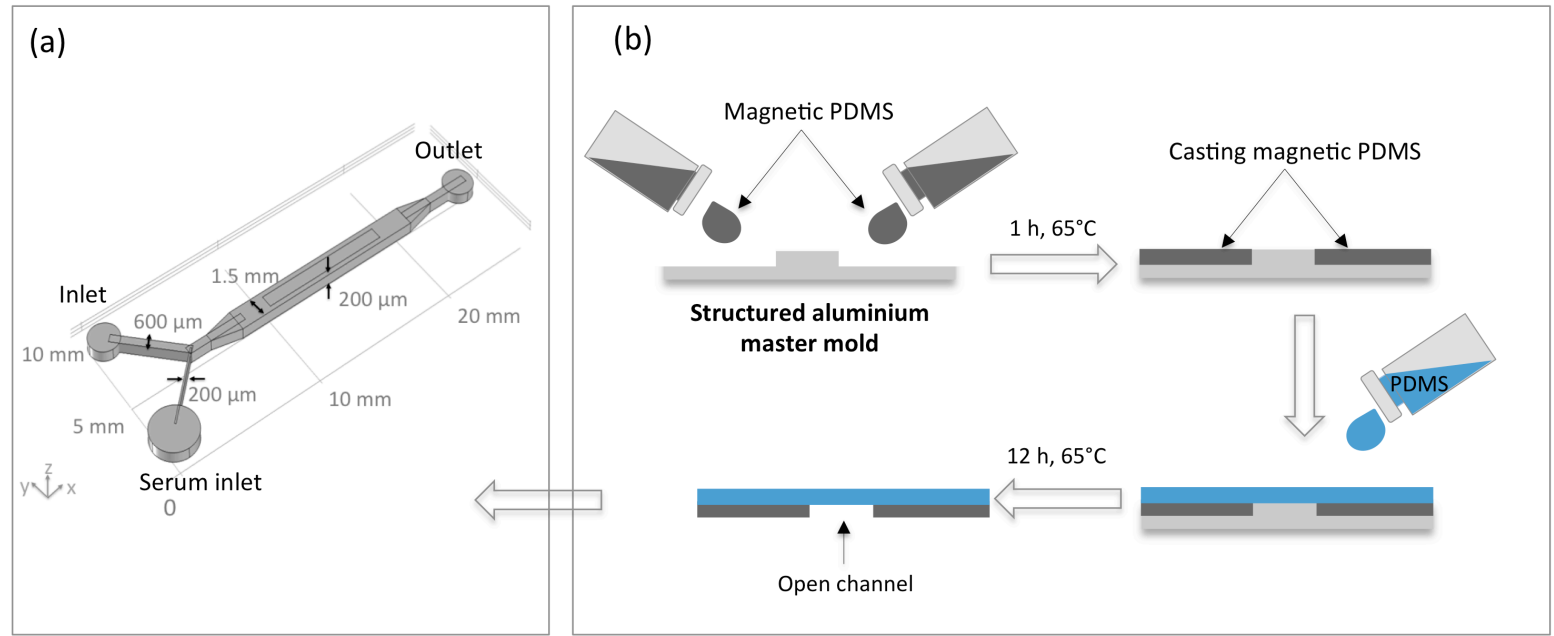

Fig 1: a) Design and b) fabrication of the magnetic microfluidic interface. 


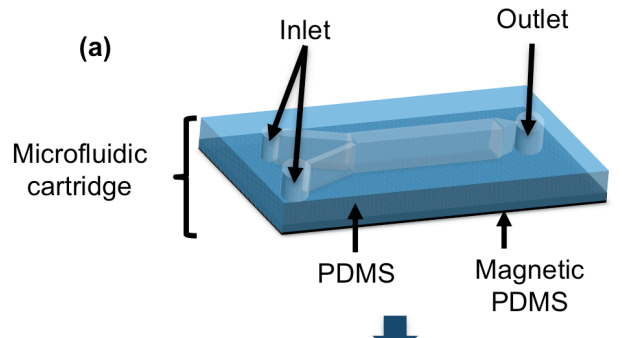

(b)
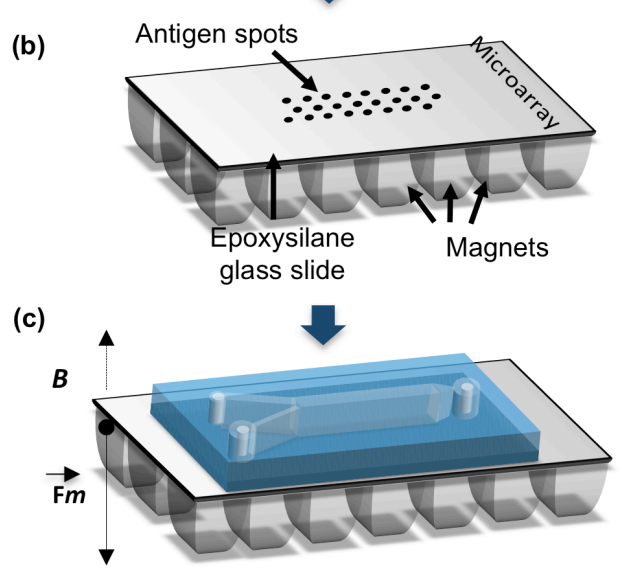
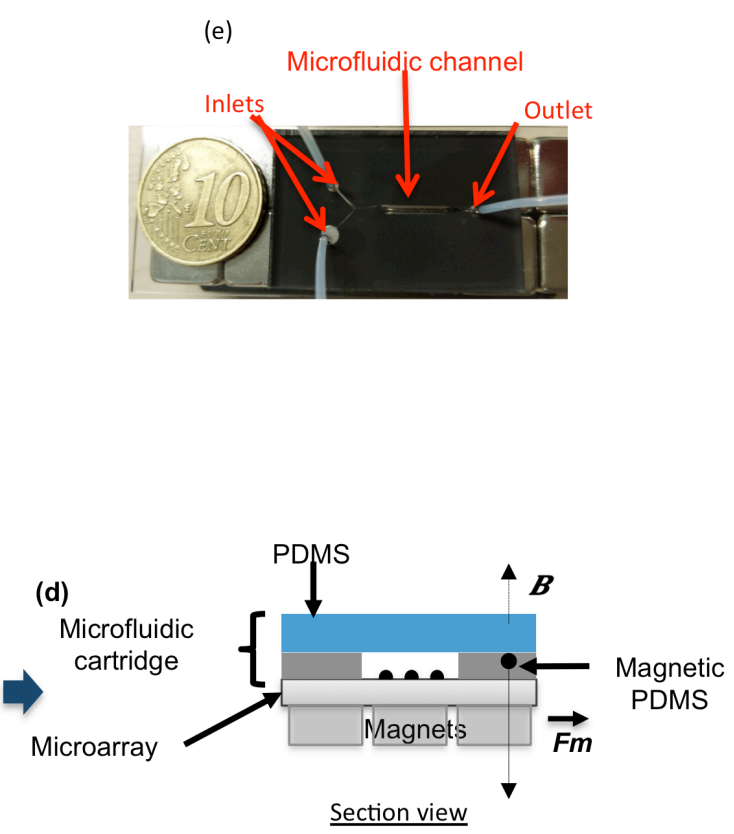

Fig 2: Schematic view of the microfluidic device. Magnetic PDMS cartridge and the microarray are hold together by magnetic force using array of magnets under the device. a) Microfluidic cartridge composed by PDMS and a magnetic PDMS layer, b) microarray composed by an epoxysilane glass slide containing an array of various antigens (85 spots), c) the microfluidic cartridge and the microarray reversibly sealed by a magnetic field to ensure a conform and hermetic contact, d) section view of the device, e) View of the microfluidic interface. 
Fig 3: Microfluidic set-up. a) passivation solution, b) washing buffer, c) solution of the secondary fluorescent antibody.

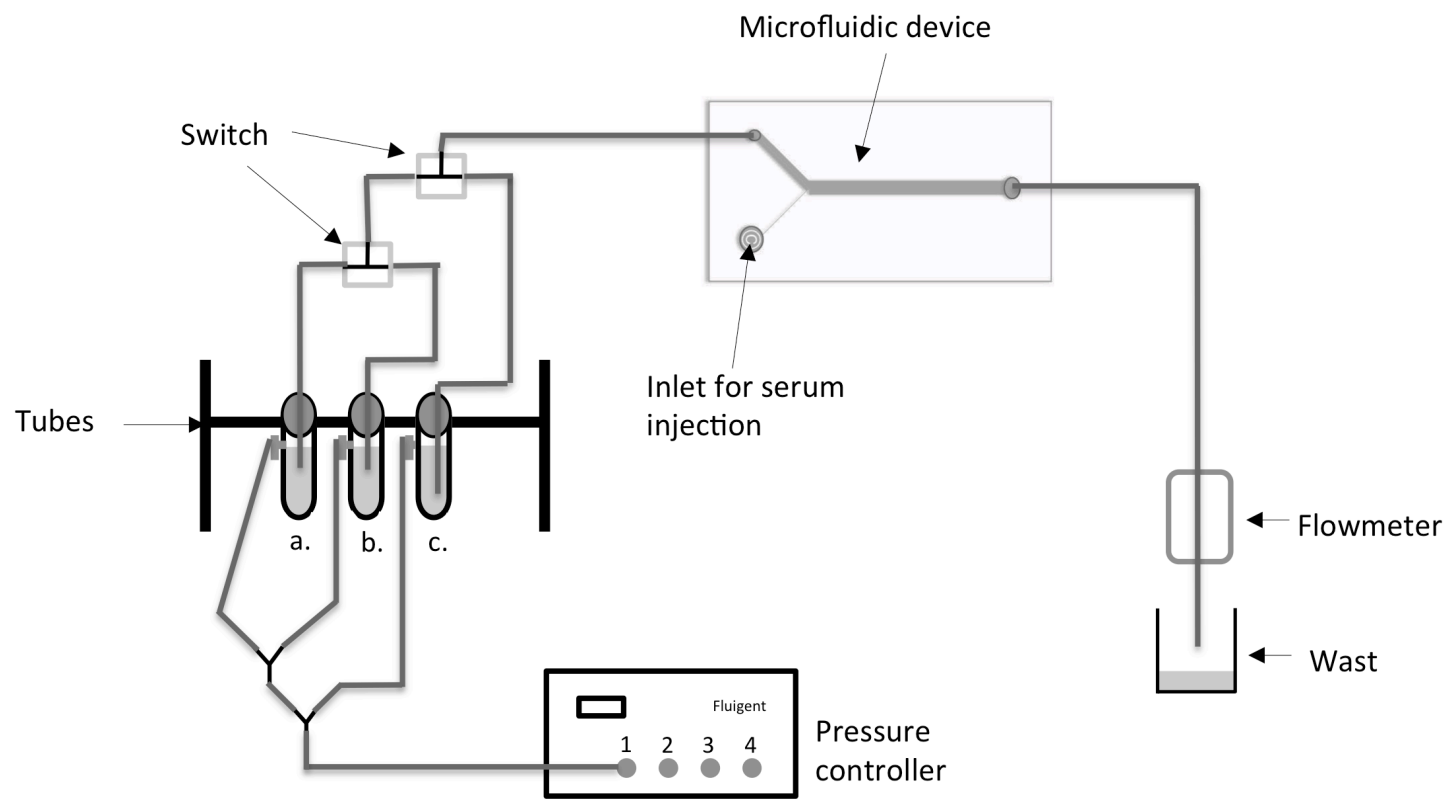

(a)

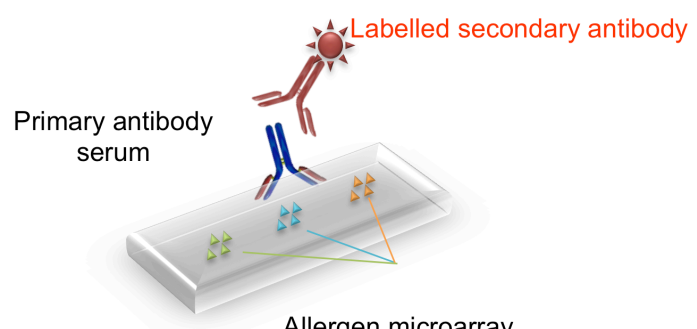

(b)

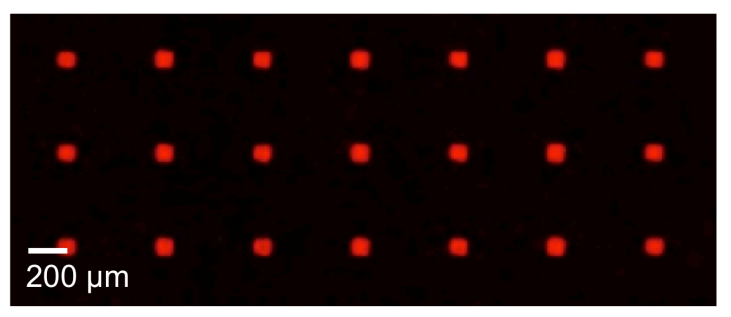

Fig 4: a) Molecular principle of the assay, b) part of microarray fluorescence image of the interacting spots after removal of the microfluidic reversible chip. 
(a)

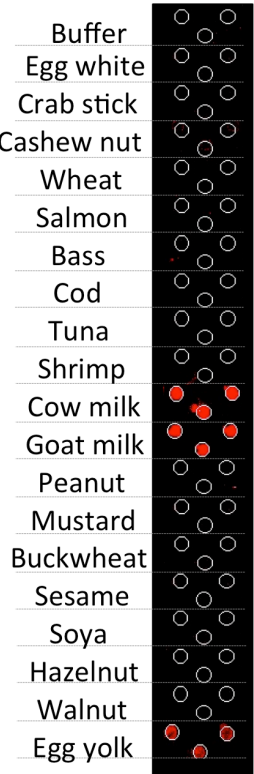

(b)

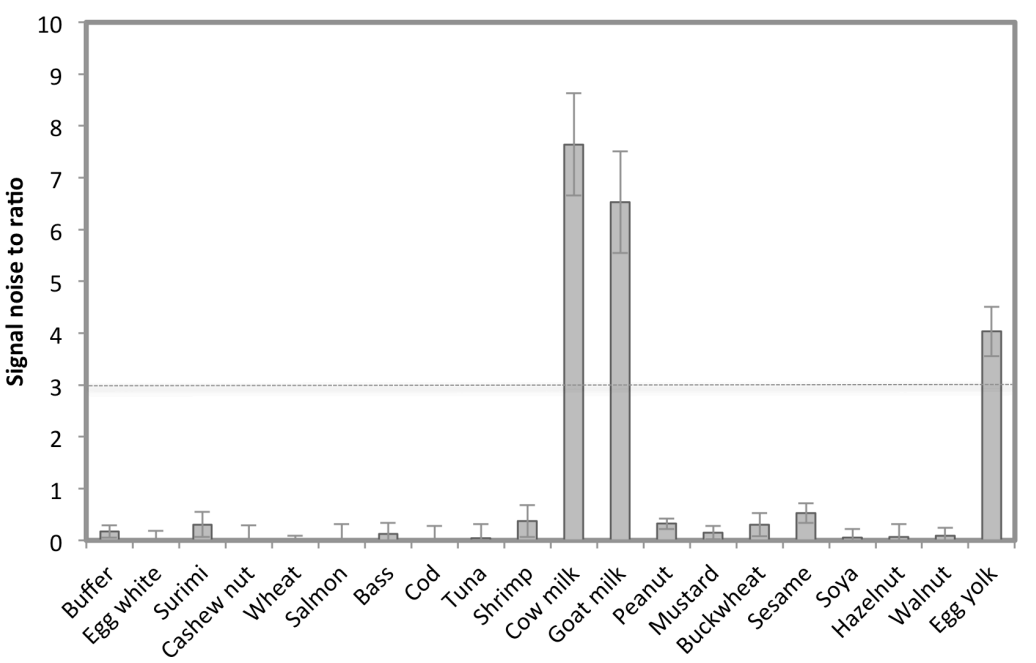

Fig 5: a) Microarray fluorescence image of the interacting spots after removal of the microfluidic reversible chip, b) signal to noise ratio quantification after food allergy immunodiagnostic using the microfluidic device. The patient was allergic to cow milk, coat milk and yolk eggs. 